\title{
Robust State Estimation of Induction Motor using Desensitized Rank Kalman Filter
}

\author{
Tai-shan Lou, Dong-xuan Han, Xiao-liang Yang, Su-xia Jiang ${ }^{1}$ \\ ${ }^{1}$ School of Electrical and Information Engineering, Zhengzhou University of Light Industry, Zhengzhou, China \\ "corresponding. jiangsx913@126.com
}

\begin{abstract}
To improve the state estimation accuracy of nonlinear induction motor with uncertain parameters, a robust desensitized rank Kalman filtering (DRKF) is proposed to reduce state estimation error sensitivities to uncertain parameters. A new sensitivity function is defined, and a novel desensitized cost function for the deterministic sampling methods is designed to obtain an optimal gain matrix. The sensitivity propagation is summarized for deterministic sampling methods. Based on the rank sample rule, the sensitivity propagation method is given, and the DRKF algorithm is derived. Two dynamic behaviors of the induction motor with two uncertain stator and rotor resistances are simulated to demonstrate that the proposed DRKF has an excellent performance.
\end{abstract}

Index Terms: Rank Kalman filter; uncertain parameter; desensitized Kalman filter; induction motor

\section{Introduction}

To reduce the cost of the induction motor and improve the reliability, some key technologies of sensorless control strategy have been developed for induction motor[1-3]. One of the essential methods is estimating the motor position and speed to feedback the closed-loop speed control, in which the state and observation system is a highly nonlinear model. One of the popular technologies for motor speed sensorless control is the nonlinear Kalman filtering (KF) class, which includes the extended Kalman filter (EKF), unscented Kalman filter (UKF), cubature Kalman filter (CKF), rank Kalman filter (RKF), ensemble Kalman filter and particle filter[4-7]. Kim et al. first used the EKF algorithm to estimate the states in the speed sensorless vector control [8], and then many variants of the EKF are successfully applied in several sensorless AC drives [2, 9, 10]. However, the EKF algorithm approximates the nonlinear function by using the Taylor series and ignoring the higher-order term. The linearization of the motor models will bring unexpected biases and decrease estimation accuracy. Then, some deterministic sample methods, such as UKF, CKF and RKF, are proposed to approximate the probability density function by using the propagation of sigma points $[4,6]$ and achieve higher accuracy in motor speed estimation [1, 11-13].

The above filtering methods can obtain optimal state estimations under the assumption that state and measurement models can be accurately modeled without any uncertainties, such as color noises, disturbances or uncertain parameters. However, the models of practical engineerings, such as an induction motor, always have model uncertainties, and the state estimation accuracy may remarkably decrease or even lead to diverging. To degrade the adverse effects of uncertain model parameters, many technologies are proposed, such as augmented filtering, consider approach[14], $H_{\infty}$ filtering[6], and desensitized filtering[15]. The desensitized filtering is proposed based on a desensitized optimal control technique, and it is used to reduce state estimation error sensitivity respected to uncertain parameters[16]. The desensitized filtering defined the sensitivity matrix and sensitivity-weighting matrix and designed a novel desensitized cost function to calculate the gain matrix, which is used to balance the state estimation and the innovation. Then, this robust strategy is expanded to a nonlinear system, and many desensitized nonlinear filtering algorithms are proposed. There are desensitized extended Kalman filter[16], desensitized divided difference filter[17], desensitized unscented Kalman filtering [18], desensitized cubature Kalman filtering [19] and desensitized ensemble new Kalman filtering [20]. Then, Lou redefined sensitivities of state estimation error and redesigned a novel desensitized cost function to obtain an analytical form solution of a gain matrix, and saved the computational time [14, 21]. Lou and Ishihara et al. respectively considered the selecting problem of the sensitivity-weighting matrix, and given some suggestions about this problem[22, 23]. Now, the desensitized filters are applied in many fields, such as Mars explore navigation[14, 17, 24], asteroid rendezvous navigation [16], hovering helicopter [19], induction motor [20, 23, 25].

This paper proposes a robust desensitized rank Kalman filter (DRKF) by introducing the desensitized optimal control method into the RKF algorithm to mitigate the sensitivities of uncertain parameters in dynamic systems. The RKF algorithm is lately proposed to solve the non-Gaussian nonlinear estimation problem by using rank statistics [7, 26]. A new desensitized cost function is modeled by using the posteriori covariance trace and a norm of the sensitivity-weighting matrix with the sensitivities. Then, an analytical gain matrix of the proposed DRKF is obtained by minimizing the above cost function to improve the accuracy of state estimation.

This article is organized as follows. Section 2 introduces the nonlinear system model, which has some uncertain parameters. Desensitized technology is briefly summarized in Section 3. Section 4 gives the sensitivity propagation of general deterministic sigma points. The robust DRKF algorithm is proposed in Section 5. Section 6 analyzes the results of numerical simulations about two dynamic behaviors of induction motor. Conclusions are shown in the end.

\section{Nonlinear system model with uncertain parameters}

Consider a discrete-time nonlinear process and measurement models with uncertain parameters and additive noises following by 


$$
\begin{aligned}
& \mathbf{x}_{k}=\mathbf{f}\left(\mathbf{x}_{k-1}, \mathbf{c}, \mathbf{u}_{k-1}\right)+\mathbf{w}_{k-1} \\
& \mathbf{z}_{k}=\mathbf{h}\left(\mathbf{x}_{k}, \mathbf{c}\right)+\mathbf{v}_{k}
\end{aligned}
$$

where $\mathbf{x}_{k} \in \mathbb{R}^{n}$ and $\mathbf{z}_{k} \in \mathbb{R}^{m}$ are the state and the measurement, respectively; functions $\mathbf{f}(\bullet)$ and $\mathbf{h}(\bullet)$ describe the nonlinear state and measurement equations, respectively; $\mathbf{c} \in \mathbb{R}^{\ell}$ is an uncertain parameter vector, and $\mathbf{u}_{k} \in \mathbb{R}^{n}$ is the control input vector. $\mathbf{w}_{k}$ is process noise and $\mathbf{v}_{k}$ is measurement noise. These two noises are independent zero-mean Gaussian processes with statistics following by

$$
\left\{\begin{array}{l}
E\left[\mathbf{w}_{k}\right]=\mathbf{0} \\
\operatorname{Cov}\left[\mathbf{w}_{k}, \mathbf{w}_{j}\right]=E\left[\mathbf{w}_{k} \mathbf{w}_{j}^{T}\right]=\boldsymbol{Q}_{k} \delta_{k j} \\
E\left[\boldsymbol{v}_{k}\right]=\mathbf{0} \\
\operatorname{Cov}\left[\boldsymbol{v}_{k}, \boldsymbol{v}_{j}\right]=E\left[\boldsymbol{v}_{k} \boldsymbol{v}_{j}^{T}\right]=\boldsymbol{R}_{k} \delta_{k j} \\
\operatorname{Cov}\left[\mathbf{w}_{k}, \boldsymbol{v}_{j}\right]=E\left[\mathbf{w}_{k} \boldsymbol{v}_{j}^{T}\right]=\mathbf{0}
\end{array}\right.
$$

where $\mathbf{Q}_{k} \in \mathbb{R}^{n \times n}$ and $\mathbf{R}_{k} \in \mathbb{R}^{m \times m}$ are all positive semi-definite matrices, $\delta_{k j}$ is Kronecker delta function.

\section{Desensitized technology}

\subsection{Sensitivity of state estimation error}

In the KF framework, the state estimation error is defined as $\tilde{\mathbf{x}}=\hat{\mathbf{x}}-\mathbf{x}$, in which $\mathbf{x}$ and $\hat{\mathbf{x}}$ are true value and state estimation of state, respectively. Under the optimality condition of the KF, which does not include color noise, perturbation or uncertain parameter, the state estimations are unbiased and satisfies $E[\tilde{\mathbf{x}}]=0$. When the model has the above uncertainties, the state estimations may be biased. Here state estimation error sensitivity respected to uncertain parameters are defined by [21]

$$
\mathbf{S}=\frac{\partial \tilde{\mathbf{x}}}{\partial \mathbf{c}}=\frac{\partial(\hat{\mathbf{x}}-\mathbf{x})}{\partial \mathbf{c}}=\frac{\partial \hat{\mathbf{x}}}{\partial \mathbf{c}}=\left(\mathbf{s}_{1}, \mathbf{s}_{2}, \cdots, \mathbf{s}_{\ell}\right)
$$

where $\mathbf{s}_{i}=\partial \tilde{\mathbf{x}} / \partial \mathbf{c}_{i}$ represents state estimation error sensitivity to the $i$ th uncertain parameter. Here, the sensitivity of true state to uncertain parameters is obviously zeros, that is to say $\partial \mathbf{x} / \partial \mathbf{c}=0$ [16].

In the KF framework, the priori state estimation error is $\tilde{\mathbf{x}}_{k}^{-} \triangleq \hat{\mathbf{x}}_{k}^{-}-\mathbf{x}_{k}$, and the posteriori estimation error is $\tilde{\mathbf{x}}_{k}^{+} \triangleq \hat{\mathbf{x}}_{k}^{+}-\mathbf{x}_{k}$. So, corresponding sensitivities of them are defined by

$$
\begin{aligned}
& \mathbf{S}_{\mathrm{k}}^{-}=\frac{\partial \tilde{\mathbf{x}}_{k}^{-}}{\partial \mathbf{c}}=\frac{\partial \hat{\mathbf{x}}_{k}^{-}}{\partial \mathbf{c}} \\
& \mathbf{S}_{\mathrm{k}}^{+}=\frac{\partial \tilde{\mathbf{x}}_{k}^{+}}{\partial \mathbf{c}}=\frac{\partial \hat{\mathbf{x}}_{k}^{+}}{\partial \mathbf{c}}
\end{aligned}
$$

where the superscripts "_." and "+" denote the priori and the posteriori, respectively. $\hat{\mathbf{x}}_{k}^{-}$and $\hat{\mathbf{x}}_{k}^{+}$are respectively the priori and the posteriori state estimation, $\mathbf{x}_{k}$ is the true state value.

Based on the computational formula of the gain matrix in the Kalman filtering framework, the relation between the priori sensitivities and the posteriori sensitivities are given by[21]

$$
\mathbf{S}_{k}^{+}=\mathbf{S}_{k}^{-}-\mathbf{K}_{k} \boldsymbol{\gamma}_{k}
$$

where

$$
\gamma_{k}=\frac{\partial \tilde{\mathbf{z}}_{k}^{-}}{\partial \mathbf{c}}=\frac{\partial \hat{\mathbf{z}}_{k}^{-}}{\partial \mathbf{c}}
$$

where $\tilde{\mathbf{z}}_{k}^{-}=\mathbf{z}_{k}-\hat{\mathbf{z}}_{k}^{-}$is the predicted measurement error, and $\hat{\mathbf{z}}_{k}^{-}$ is the predicted measurement.

Note that the sensitivity of the gain matrix is $\partial \mathbf{K}_{k} / \partial \mathbf{c}=0$, and the details can be seen in the reference [16, $18]$.

\subsection{Desensitized cost function and gain matrix}

The core idea of the desensitized technology is using the penalty function to penalize the original cost function of the KF algorithm, and the penalty function is a weighted norm of the sensitivities of the state estimation error respected to uncertain parameters. The desensitized cost function is given by [21]

$$
J_{d}=\operatorname{Tr}\left(\mathbf{P}_{k}^{+}\right)+\operatorname{Tr}\left(\mathbf{S}_{k}^{+} W_{k} \mathbf{S}_{k}^{+T}\right)
$$

where " $T r$ " is the trace of a matrix, $\mathbf{P}_{k}^{+}$is the posteriori covariance, $W_{k} \in \mathbb{R}^{\ell \times \ell}$ is a semi-definite diagonal matrix.

Substituting expression of covariance $\mathbf{P}_{k}^{+}=\mathbf{P}_{x x, k}^{-}-\mathbf{P}_{x z, k} \mathbf{K}_{k}^{T}-\mathbf{K}_{k} \mathbf{P}_{x z, k}^{T}+\mathbf{K}_{k} \mathbf{P}_{z z, k} \mathbf{K}_{k}^{T}$ and $\mathbf{S}_{k}^{+}$in Eq.(7) into Eq.(9), and taking partial derivation of $J_{d}$ respected to $\mathbf{K}_{k}$ gives by

$$
\frac{\partial J_{d}}{\partial \mathbf{K}_{k}}=2 \mathbf{K}_{k} \mathbf{P}_{z z, k}-2 \mathbf{P}_{x z, k}-2 \mathbf{S}_{k}^{-} \mathbf{W} \boldsymbol{\gamma}_{k}^{T}+2 \mathbf{K}_{k} \boldsymbol{\gamma}_{k} \mathbf{W} \boldsymbol{\gamma}_{k}^{T}
$$

Setting $\partial J_{d} / \partial \mathbf{K}_{k}=0$ obtains

$$
\mathbf{K}_{k}=\left(\mathbf{P}_{x z, k}+\mathbf{S}_{k}^{-} \mathbf{W}_{k} \boldsymbol{\gamma}_{k}^{T}\right)\left(\mathbf{P}_{z z, k}+\boldsymbol{\gamma}_{k} \mathbf{W}_{k} \boldsymbol{\gamma}_{k}^{T}\right)^{-1}
$$

Note that this is a general formula for the DKF framework, which includes the KF, EKF, UKF and their variants.

\section{Sensitivity propagation of deterministic sigma points}

The desensitized technology needs the sensitivities of the state estimation error to compose the penalty function. So, the calculation method of the sensitivities is essential for the desensitized cost function. For the KF and EKF, the sensitivities are calculated by the sensitivity propagation function, which can be obtained by the Jacobian matrix of the process function and measurement function respected to uncertain parameters [16]. For the deterministic sample Kalman filtering class, which includes the UKF, CKF, and CDKF, their sensitivities should be propagated by the sigma points $[18,19,25]$. Here, we summarized the sensitivities of the sigma points for the general deterministic sample filtering method.

Assuming that the sigma points are given by

$$
\chi_{j}=\overline{\mathbf{x}}+\xi\left(\sqrt{\mathbf{P}_{x}}\right)_{j}, j=1, \cdots, L
$$

where $\left(\sqrt{\mathbf{P}_{x}}\right)_{j}$ is the $j$ th column of $\sqrt{\mathbf{P}_{x}} ; \xi$ is weighting coefficient; $L$ is the number of sigma points.

The transformed set of sigma points are calculated by

$$
\chi_{j}^{*}=\mathbf{f}\left(\chi_{j}, \overline{\mathbf{c}}, \mathbf{u}\right), j=1, \cdots, L
$$

The corresponding mean, covariance and cross-covariance are calculated by

$$
\begin{aligned}
\hat{\mathbf{x}} & =\sum_{j=1}^{L} w_{j}^{m} \boldsymbol{\chi}_{j}^{*} \\
\mathbf{P}_{\hat{x} \hat{x}} & =\sum_{j=1}^{L} w_{j}^{\mathrm{c}}\left(\chi_{j}^{*}-\hat{\mathbf{x}}\right)\left(\chi_{j}^{*}-\hat{\mathbf{x}}\right)^{T}
\end{aligned}
$$




$$
\mathbf{P}_{x \hat{x}}=\sum_{j=1}^{L} w_{j}^{c}\left(\chi_{j}^{*}-\overline{\mathbf{x}}\right)\left(\chi_{j}^{*}-\hat{\mathbf{x}}\right)^{T}
$$

where, $w_{j}^{m}$ and $w_{j}^{c}$ are the weights of the $j$ th sigma point, and the superscript $m$ denotes mean value, $c$ denotes covariance.

Based on the above formulas, the sensitivities propagated by sigma points are given by

$$
\frac{\partial \boldsymbol{\chi}_{j}}{\partial \mathbf{c}}=\frac{\partial \mathbf{x}}{\partial \mathbf{c}}+\xi \frac{\partial\left(\sqrt{\mathbf{P}_{x}}\right)_{j}}{\partial \mathbf{c}}, j=1, \cdots, L
$$

where $\partial \overline{\mathbf{x}} / \partial \mathbf{c}$ and $\partial\left(\sqrt{\mathbf{P}_{x}}\right)_{j} / \partial \mathbf{c}$ are the previous sensitivities of state estimation and $\sqrt{\mathbf{P}_{x}}$, respectively.

The transformed set of the sensitivities of sigma points are evaluated by

$$
\frac{\partial \boldsymbol{\chi}_{j}^{*}}{\partial \mathbf{c}}=\left.\frac{\partial \mathbf{f}\left(\chi_{j}, \overline{\mathbf{c}}, \mathbf{u}\right)}{\partial \boldsymbol{\chi}_{j}} \frac{\partial \boldsymbol{\chi}_{j}}{\partial \mathbf{c}}\right|_{\mathbf{c}=\overline{\mathbf{c}}}+\left.\frac{\partial \mathbf{f}\left(\boldsymbol{\chi}_{j}, \mathbf{c}, \mathbf{u}\right)}{\partial \mathbf{c}}\right|_{\mathbf{c}=\overline{\mathbf{c}}}
$$

The sensitivity of the state estimation error is

$$
\mathbf{S}=\frac{\partial(\hat{\mathbf{x}}-\mathbf{x})}{\partial \mathbf{c}}=\sum_{j=1}^{L} w_{j}^{m} \frac{\partial \boldsymbol{\chi}_{j}^{*}}{\partial \mathbf{c}}
$$

The sensitivity of covariance and cross-covariance are following by

$$
\begin{gathered}
\frac{\partial \mathbf{P}_{\hat{x} \hat{x}}}{\partial \mathbf{c}}=\sum_{j=1}^{L} w_{j}^{c}\left[\left(\frac{\partial \boldsymbol{\chi}_{j}^{*}}{\partial \mathbf{c}}-\mathbf{S}\right)\left(\chi_{j}^{*}-\hat{\mathbf{x}}\right)^{T}+\left(\chi_{j}^{*}-\hat{\mathbf{x}}\right)\left(\frac{\partial \boldsymbol{\chi}_{j}^{*}}{\partial \mathbf{c}}-\mathbf{S}\right)^{T}\right] \\
\frac{\partial \mathbf{P}_{x \hat{x}}}{\partial \mathbf{c}}=\sum_{j=1}^{L} w_{j}^{c}\left[\left(\frac{\partial \boldsymbol{\chi}_{j}^{*}}{\partial \mathbf{c}}-\frac{\partial \overline{\mathbf{x}}}{\partial \mathbf{c}}\right)\left(\chi_{j}^{*}-\hat{\mathbf{x}}\right)^{T}+\left(\chi_{j}^{*}-\overline{\mathbf{x}}\right)\left(\frac{\partial \chi_{j}^{*}}{\partial \mathbf{c}}-\mathbf{S}\right)^{T}\right]
\end{gathered}
$$

Remark 1: The detail of computation of the sensitivity of the square-root matrix $\sqrt{\mathbf{P}}$ is in references [19, 21].

\section{ROBUST DRKF ALGORITHM}

The core idea of the RKF algorithm is the rank sample method, which is based on the rank statistic. The distribution of sigma points depends on the quantile of the median rank and the layer number of sampling. Based on the symmetrical distribution and two-sample layer, the sigma point set is given by

$$
\boldsymbol{\chi}_{j}=g\left(\overline{\mathbf{x}}, \sqrt{\mathbf{P}_{x}}\right)= \begin{cases}\overline{\mathbf{x}}+u_{p_{1}}\left(\sqrt{\mathbf{P}_{x}}\right)_{j} & j=1, \cdots, n \\ \overline{\mathbf{x}}-u_{p_{1}}\left(\sqrt{\mathbf{P}_{x}}\right)_{j-n} & j=n+1, \cdots, 2 n \\ \overline{\mathbf{x}}+u_{p_{2}}\left(\sqrt{\mathbf{P}_{x}}\right)_{j-2 n} & j=2 n+1, \cdots, 3 n \\ \overline{\mathbf{x}}-u_{p_{2}}\left(\sqrt{\mathbf{P}_{x}}\right)_{j-3 n} & j=3 n+1, \cdots, 4 n\end{cases}
$$

where $u_{p_{j}}$ is the standard normal deviator, which is calculated by the median rank $p_{j}=(j+2.7) / 5.4$, and their values are respectively $p_{1}=0.6852, u_{p_{1}}=0.4823$ and $p_{2}=0.8704$, $u_{p_{2}}=1.1283$.

Based on the above rank sample method and desensitized technology, the robust DRKF algorithm is derived and summarized as follows.

Step 1: Initializing the state $\hat{\mathbf{x}}_{0}$, the error covariance matrix $\mathbf{P}_{0}$, the sensitivity-weighting matrix $\mathbf{W}$, the sensitivity matrix $\mathbf{S}_{0}$, and sensitivity covariance matrix $\partial \mathbf{P}_{0} / \partial \mathbf{c}$.

Step 2: Calculating the propagated sigma points of the state

$$
\chi_{j, k-1}=g\left(\hat{\mathbf{x}}_{k-1}^{+}, \sqrt{\mathbf{P}_{k-1}^{+}}\right)
$$

where $\hat{\mathbf{x}}_{k-1}^{+}$and $\mathbf{P}_{k-1}^{+}$are the state estimation and the error covariance at a time step $k-1$, respectively.

Calculating the propagated sensitivities of sigma points following by

$$
\frac{\partial \boldsymbol{\chi}_{j, k-1}}{\partial \mathbf{c}}=g\left(\mathbf{S}_{k-1}^{+}, \frac{\partial \sqrt{\mathbf{P}_{k-1}^{+}}}{\partial \mathbf{c}}\right)
$$

Step 3: Evaluating the predicted sigma points

$$
\chi_{j, k}^{*}=\mathbf{f}\left(\chi_{j, k-1}, \overline{\mathbf{c}}, \mathbf{u}_{k-1}\right), \quad j=1,2, \cdots, 4 n
$$

and computing the sensitivities of the predicted sigma points

$$
\frac{\partial \chi_{j, k}^{*}}{\partial \mathbf{c}}=\left.\frac{\partial \mathbf{f}\left(\boldsymbol{\chi}_{j, k-1}, \overline{\mathbf{c}}, \mathbf{u}_{k-1}\right)}{\partial \boldsymbol{\chi}_{j, k-1}} \frac{\partial \boldsymbol{\chi}_{j, k-1}}{\partial \mathbf{c}}\right|_{\mathbf{c}=\overline{\mathbf{c}}}+\left.\frac{\partial \mathbf{f}\left(\boldsymbol{\chi}_{j, k-1}, \mathbf{c}, \mathbf{u}_{k-1}\right)}{\partial \mathbf{c}}\right|_{\mathbf{c}=\overline{\mathbf{c}}}
$$

Step 4: Calculating the priori state

$$
\hat{\mathbf{x}}_{k}^{-}=\frac{1}{4 n} \sum_{j=1}^{4 n} \chi_{j, k}^{*}
$$

and evaluating the error covariance matrix

$$
\mathbf{P}_{x x, k}^{-}=\frac{1}{\omega} \sum_{j=1}^{4 \mathrm{n}}\left\{\left(\chi_{j, k}^{*}-\hat{\mathbf{x}}_{k}^{-}\right)\left(\chi_{j, k}^{*}-\hat{\mathbf{x}}_{k}^{-}\right)^{T}\right\}+\boldsymbol{Q}_{k-1}
$$

where $\omega$ is the covariance weighting coefficient, which is calculated by $\omega=2\left(u_{p_{1}}^{2}+u_{p_{2}}^{2}\right)$.

Then, the corresponding sensitivities of the priori state and covariance are given by

$$
\begin{gathered}
\mathbf{S}_{k}^{-}=\frac{\partial \hat{\mathbf{x}}_{k}^{-}}{\partial \mathbf{c}}=\frac{1}{4 n} \sum_{j=1}^{4 n} \frac{\partial \boldsymbol{\chi}_{j, k}^{*}}{\partial \mathbf{c}} \\
\frac{\partial \mathbf{P}_{x, k}^{-}}{\partial \mathbf{c}}=\frac{1}{\omega} \sum_{j=1}^{4 n}\left\{\left(\frac{\partial \chi_{j, k}^{*}}{\partial \mathbf{c}}-\mathbf{S}_{k}^{-}\right)\left(\chi_{j, k}^{*}-\hat{\mathbf{x}}_{k}^{-}\right)^{T}+\left(\chi_{j, k}^{*}-\hat{\mathbf{x}}_{k}^{-}\right)\left(\frac{\partial \chi_{j, k}^{*}}{\partial \mathbf{c}}-\mathbf{S}_{k}^{-}\right)^{T}\right\}
\end{gathered}
$$

Step 5: Resampling sigma points

$$
\bar{\chi}_{j, k}=g\left(\hat{\mathbf{x}}_{k}^{-}, \sqrt{\mathbf{P}_{x x, k}^{-}}\right)
$$

and computing the sensitivities of new sigma points

$$
\frac{\partial \overline{\boldsymbol{\chi}}_{j, k}}{\partial \mathbf{c}}=g\left(\mathbf{S}_{k}^{-}, \frac{\partial \sqrt{\mathbf{P}_{x, k}^{-}}}{\partial \mathbf{c}}\right)
$$

Step 6: Evaluating the predicted measurement using the sigma points

$$
\mathbf{Z}_{j, k}=\mathbf{h}\left(\bar{\chi}_{j, k}, \overline{\mathbf{c}}\right), \quad j=1,2, \cdots, 4 n
$$

and propagating the sensitivities of the predicted measurement points

$$
\frac{\partial \mathbf{Z}_{j, k}}{\partial \mathbf{c}}=\left.\frac{\partial \mathbf{h}\left(\overline{\boldsymbol{\chi}}_{j, k}, \overline{\mathbf{c}}\right)}{\partial \overline{\boldsymbol{\chi}}_{j, k}} \frac{\partial \overline{\boldsymbol{\chi}}_{j, k}}{\partial \mathbf{c}}\right|_{\mathbf{c}=\overline{\mathbf{c}}}+\left.\frac{\partial \mathbf{h}\left(\overline{\boldsymbol{\chi}}_{j, k}, \mathbf{c}\right)}{\partial \mathbf{c}}\right|_{\mathbf{c}=\overline{\mathbf{c}}}
$$

Step 7: Estimating the priori measurement

$$
\hat{\mathbf{z}}_{k}^{-}=\frac{1}{4 n} \sum_{j=1}^{4 n} \mathbf{Z}_{j, k}
$$

and calculating the sensitivities of the priori measurement

$$
\boldsymbol{\gamma}_{k}=\frac{\partial \hat{\mathbf{z}}_{k}^{-}}{\partial \mathbf{c}}=\frac{1}{4 n} \sum_{j=1}^{4 n} \frac{\partial \mathbf{Z}_{j, k}}{\partial \mathbf{c}}
$$

Step 8: Evaluating the innovation covariance matrix and the cross-covariance matrix

$$
\mathbf{P}_{z z, k}=\frac{1}{\omega} \sum_{j=1}^{4 n}\left\{\left(\mathbf{Z}_{j, k}-\hat{\mathbf{z}}_{k}^{-}\right)\left(\mathbf{Z}_{j, k}-\hat{\mathbf{z}}_{k}^{-}\right)^{T}\right\}+\boldsymbol{R}_{k}
$$




$$
\mathbf{P}_{x z, k}=\frac{1}{\omega} \sum_{j=1}^{4 n}\left\{\left(\bar{\chi}_{j, k}-\hat{\mathbf{x}}_{k}^{-}\right)\left(\mathbf{Z}_{j, k}-\hat{\mathbf{z}}_{k}^{-}\right)^{T}\right\}
$$

and calculating the sensitivities of two covariance matrices

$$
\begin{gathered}
\frac{\partial \mathbf{P}_{z z, k}}{\partial \mathbf{c}}=\frac{1}{\omega} \sum_{j=1}^{4 n}\left\{\left(\frac{\partial \mathbf{Z}_{j, k}}{\partial \mathbf{c}}-\boldsymbol{\gamma}_{k}\right)\left(\mathbf{Z}_{j, k}-\hat{\mathbf{z}}_{k}^{-}\right)^{T}+\left(\mathbf{Z}_{j, k}-\hat{\mathbf{z}}_{k}^{-}\right)\left(\frac{\partial \mathbf{Z}_{j, k}}{\partial \mathbf{c}}-\boldsymbol{\gamma}_{k}\right)^{T}\right\} \\
\frac{\partial \mathbf{P}_{x z, k}}{\partial \mathbf{c}}=\frac{1}{\omega} \sum_{j=1}^{4 n}\left\{\left(\frac{\partial \overline{\boldsymbol{\chi}}_{j, k}}{\partial \mathbf{c}}-\mathbf{S}_{k}^{-}\right)\left(\mathbf{Z}_{j, k}-\hat{\mathbf{z}}_{k}^{-}\right)^{T}+\left(\overline{\boldsymbol{\chi}}_{j, k}-\hat{\mathbf{x}}_{k}^{-}\right)\left(\frac{\partial \mathbf{Z}_{j, k}}{\partial \mathbf{c}}-\boldsymbol{\gamma}_{k}\right)^{T}\right\}
\end{gathered}
$$

Step 9: Computing the gain matrix

$$
\mathbf{K}_{k}=\left(\mathbf{P}_{x z, k}+\mathbf{S}_{k}^{-} \mathbf{W}_{k} \boldsymbol{\gamma}_{k}^{T}\right)\left(\mathbf{P}_{z z, k}+\boldsymbol{\gamma}_{k} \mathbf{W}_{k} \boldsymbol{\gamma}_{k}^{T}\right)^{-1}
$$

Step 10: Estimating the posteriori state

$$
\hat{\mathbf{x}}_{k}^{+}=\hat{\mathbf{x}}_{k}^{-}+\mathbf{K}_{k}\left(\mathbf{z}_{k}-\hat{\mathbf{z}}_{k}^{-}\right)
$$

and estimating the posteriori covariance matrix

$$
\mathbf{P}_{k}^{+}=\mathbf{P}_{x x, k}^{-}-\mathbf{P}_{x z, k} \mathbf{K}_{k}^{T}-\mathbf{K}_{k} \mathbf{P}_{x z, k}^{T}+\mathbf{K}_{k} \mathbf{P}_{z z, k} \mathbf{K}_{k}^{T}
$$

Then, sensitivities of the posteriori state and posteriori covariance are given respectively by

$$
\begin{gathered}
\mathbf{S}_{k}^{+}=\mathbf{S}_{k}^{-}-\mathbf{K}_{k} \boldsymbol{\gamma}_{k} \\
\frac{\partial \mathbf{P}_{k}^{+}}{\partial \mathbf{c}}=\frac{\partial \mathbf{P}_{x x, k}^{-}}{\partial \mathbf{c}}-\frac{\partial \mathbf{P}_{x z, k}}{\partial \mathbf{c}} \mathbf{K}_{k}^{T}-\mathbf{K}_{k} \frac{\partial \mathbf{P}_{x z, k}^{T}}{\partial \mathbf{c}}+\mathbf{K}_{k} \frac{\partial \mathbf{P}_{z z, k}}{\partial \mathbf{c}} \mathbf{K}_{k}^{T}
\end{gathered}
$$

\section{Simulation}

To verify the performance of the novel robust DRKF, an induction motor with uncertain stator and rotor resistances are considered in simulation $[20,25]$. The results of the DRKF with the results of the perfect RKF (perf. RKF) and imperfect RKF (imp. RKF) algorithms are selected to compare by using the root mean squared errors (RMSEs). The "perf. RKF" means that parameters are precisely known, and the "imp. RKF" denotes that the values of parameters come from the previous experience.

In the stationary $\alpha-\beta$ reference frame, discrete state equation of induction motor is modeled by

Table 1

Parameters of the induction motor system in simulation

\begin{tabular}{cc}
\hline \hline Parameter & Value \\
\hline Number of pole pairs & $p_{n}=2$ \\
mutual inductance & $L_{m}=0.253 \mathrm{H}$ \\
stator inductance & $L_{s}=0.265 \mathrm{H}$ \\
rotor inductance & $L_{r}=0.265 \mathrm{H}$ \\
supply voltage & $U=220 \mathrm{~V}$ \\
\hline \hline
\end{tabular}

$$
\begin{aligned}
& \mathbf{x}_{k}=\mathbf{f}\left(\mathbf{x}_{k-1}, \mathbf{c}, \mathbf{u}_{k-1}\right)+\mathbf{w}_{k} \\
& {\left[\begin{array}{l}
\mathrm{x}_{1_{k-1}}+d t\left(-\frac{\mathbf{c}_{1} L_{r}^{2}+\mathbf{c}_{2} L_{m}{ }^{2}}{\sigma L_{s} L_{r}^{2}} x_{1_{k-1}}+\frac{L_{m}}{\sigma L_{s} L_{r} T_{r}} x_{3_{k-1}}+\frac{L_{m}}{\sigma L_{s} L_{r}} x_{5_{k-1}} x_{4_{k-1}}+\frac{u_{1_{k-1}}}{\sigma L_{s}}\right) \\
\mathrm{x}_{2_{k-1}}+d t\left(-\frac{\mathbf{c}_{1} L_{r}^{2}+\mathbf{c}_{2} L_{m}^{2}}{\sigma L_{s} L_{r}^{2}} x_{2_{k-1}}+\frac{L_{m}}{\sigma L_{s} L_{r} T_{r}} x_{4_{k-1}}-\frac{L_{m}}{\sigma L_{s} L_{r}} x_{5_{k-1}} x_{3_{k-1}}+\frac{u_{2_{k-1}}}{\sigma L_{s}}\right) \\
x_{3_{k-1}}+d t\left(\frac{L_{m}}{T_{r}} x_{1_{k-1}}-\frac{1}{T_{r}} x_{3_{k-1}}-x_{5_{k-1}} x_{4_{k-1}}\right) \\
x_{4_{k-1}}+d t\left(\frac{L_{m}}{T_{r}} x_{2_{k-1}}-\frac{1}{T_{r}} x_{4_{k-1}}+x_{5_{k-1}} x_{3_{k-1}}\right) \\
x_{5_{k-1}}+d t\left(\frac{p_{n}^{2} L_{m}}{J L_{r}}\left(x_{2_{k-1}} x_{3_{k-1}}-x_{1_{k-1}} x_{4_{k-1}}\right)-\frac{p_{n}}{J} T_{L}\right)
\end{array}\right]+\mathbf{w}}
\end{aligned}
$$

where $\mathbf{x}_{k}=\left[x_{1, k}, x_{2, k}, x_{3, k}, x_{4, k}, x_{5, k}\right]^{T}$, in which $x_{1, k}$ is the first stator current, $x_{2, k}$ is the second stator current, $x_{3, k}$ is the first rotor flux, $x_{4, k}$ is the second rotor flux, $x_{5, k}$ is the angular velocity; $\mathrm{T}_{L}$ is the load torque, $J$ is the rotor moment of inertia, $p_{n}$ is the number of pole-pairs, $T_{r}$ is the rotor time constant acquired by $\mathrm{T}_{r}=L_{r} / R_{r}, \sigma$ is the no-load magnetic leakage factor of motor, $L_{s}$ and $L_{r}$ respectively are the stator winding inductance and rotor winding inductance, and $L_{m}$ is mutual inductance between stator and rotor; $u_{1, k}$ is the first stator voltage control input at time step $k, u_{2, k}$ is the second stator voltage control input; $d t$ is the sampling period; $\mathbf{c}=\left[R_{s}, R_{r}\right]^{T}$ is the uncertain parameter vector, in which $R_{s}$ and $R_{r}$ are the stator resistance and rotor resistance, respectively; $\mathbf{w}_{k}$ is a white Gaussian noise with zero mean and covariance $\mathbf{Q}_{k}$.

The measurements are the first stator current $x_{1_{k}}$ and the first rotor flux $x_{3_{k}}$ of the induction motor, and the measurement equation of the induction motor system is modeled by

$$
\mathbf{z}_{k}=\mathbf{H}_{k} \mathbf{x}_{k}+\mathbf{v}_{k}
$$

where $\mathbf{H}_{k}$ is the observation matrix, satisfies

$$
\mathbf{H}_{k}=\left[\begin{array}{lllll}
1 & 0 & 0 & 0 & 0 \\
0 & 0 & 1 & 0 & 0
\end{array}\right]
$$

where $\mathbf{v}_{k}$ is white Gaussian noise with zero mean with covariance $\mathbf{R}_{k}$, which is independent with the process noise $\mathbf{w}_{k}$.

For the above system, the stator resistance and the rotor resistance may vary with different working conditions and the environment in the actual motor operation process. The stator resistance only has a relation with two stator currents. The rotor resistance has relations with two stator currents and two rotor fluxes. Therefore, the state estimation accuracy of the induction motor is sensitivity to the above two varying motor parameters. In this simulation, we assume that the stator resistance and the rotor resistance distribute to the uniform distribution $U\left(0.75 \bar{R}_{s}, 1.25 \bar{R}_{s}\right)$ and $U\left(0.75 \bar{R}_{r}, 1.25 \bar{R}_{r}\right)$, respectively. Their reference values are $\bar{R}_{s}=2.68 \Omega$ and $\bar{R}_{r}=2.85 \Omega$, respectively. 
The sensitivity-weighting matrices are given by $W_{1}=7.1824$ and $W_{2}=8.1225$ in simulation.

The initial state $\mathbf{x}_{0}=[0,0,0,0,0]^{T}$, the initial covariance $\mathbf{P}_{0}=\operatorname{diag}(10,10,20,20,50)$. The covariance of the process and measurement noises are $\mathbf{Q}_{k}=\operatorname{diag}\left(10^{-2}, 10^{-2}, 10^{-4}, 10^{-4}, 1\right)$ and $\mathbf{R}_{k}=\operatorname{diag}\left(10^{-2}, 10^{-4}\right)$, respectively. The sampling period $d t=10^{-3} s$ in simulation.

Two dynamic behaviors of induction motor are simulated, which are the ideal no-load start and three-phase sudden short circuit fault. The values of the parameters of the induction motor system in the simulation are shown in Table 1.

\subsection{Ideal no-load start at rated voltage}

The induction motor is in a static state before starting, and $\mathrm{T}_{L}=0$. Figure 1 shows the true values of the states and state estimations of three filters for one simulation. It can be seen that the rotation speed of the three-phase induction motor quickly has a peak value in the process of starting, and then tend to be stable. The same are the currents and rotor fluxes.

Figure 1 shows the RMSEs of three filters for the process of ideal no-load start at rated voltage. During the process of ideal no-load start, the induction system model has uncertainties on the stator resistance and rotor resistance. As can be seen from Fig.2, the RMSEs of the perf. RKF is always the smallest because the true values of the stator resistance and rotor

resistance are known. The RMSEs of the DRKF is obviously smaller than that of the imp. RKF.

Figures 3-4 show that the state sensitivities with respect to the uncertain stator and rotor resistances for the ideal no-load start at rated voltage. Compared to the imp.RKF, the proposed DRKF has smaller sensitivities to two resistances.

\subsection{Three-phase sudden short circuit fault}

Assuming the motor has been running in the ideal no-load state, then a sudden short circuit fault, which is from $220 \mathrm{~V}$ to 0 in $0.12 \mathrm{~s}$ moment, and from 0 to $220 \mathrm{~V}$ in $0.22 \mathrm{~s}$ moment, happens. Figure 5 shows the true values of the states and state estimations of three filters for one simulation. From Fig. 5, we can see that a three-phase symmetric short circuit fault occurs at the stator terminal at $0.12 \mathrm{~s}$, and the stator current and rotor flux are affected. After the fault was repaired at $0.22 \mathrm{~s}$, the motor terminal voltage suddenly recovered, the stator current and rotor flux generated a specific impact, which rose to the maximum value in an instant, then gradually decreased to the stable value, the speed rapidly rose to the stable value, and the motor returned to the ideal no-load running state.

According to Fig.6, the DRKF algorithm also has a better performance than that of the imp. RKF as a whole when the three-phase sudden short circuit fault happens at $0.12 \mathrm{~s}-0.22 \mathrm{~s}$.

From the above two conditions of the induction motor, we can see that the proposed DRKF algorithm can mitigate the adverse effects of the uncertain stator and rotor resistances, and has a better performance than the imp. RKF.
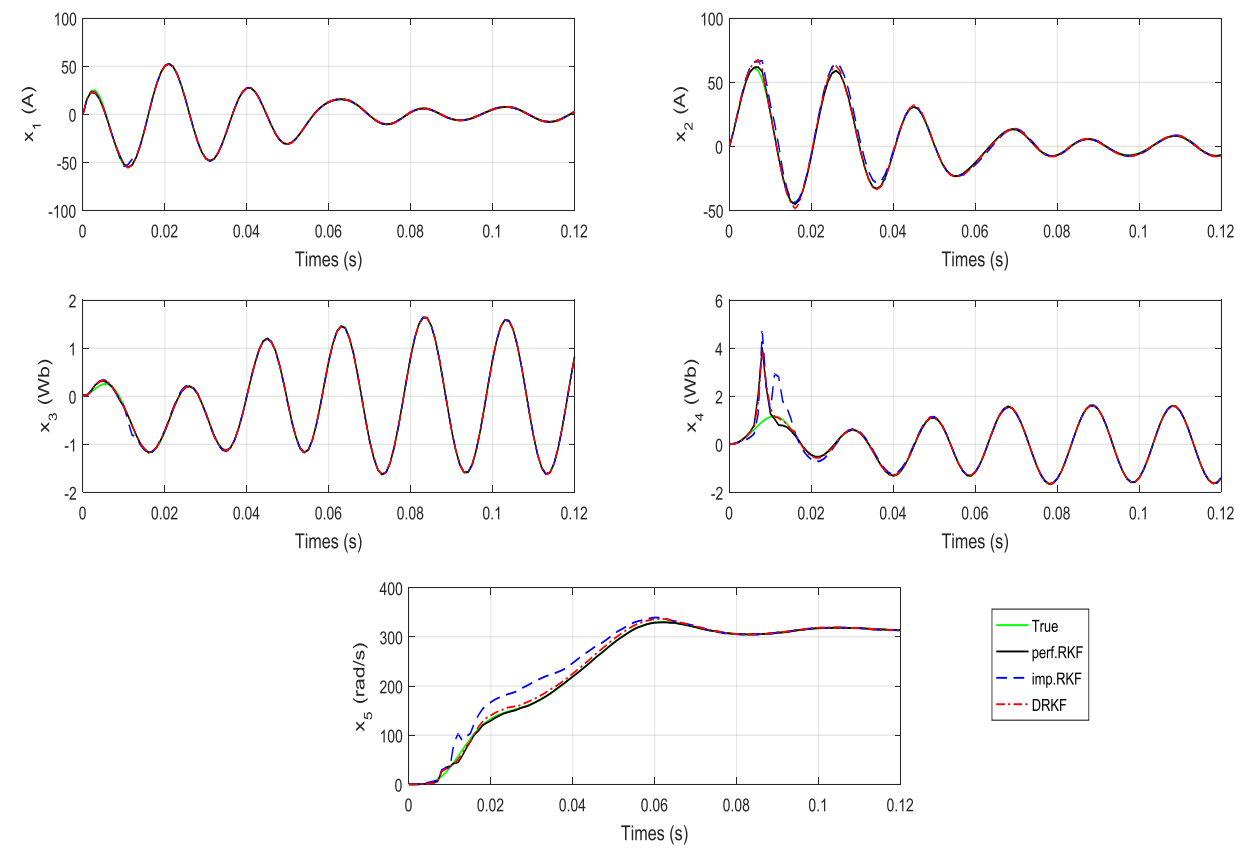

Fig. 1. True values and state estimations 

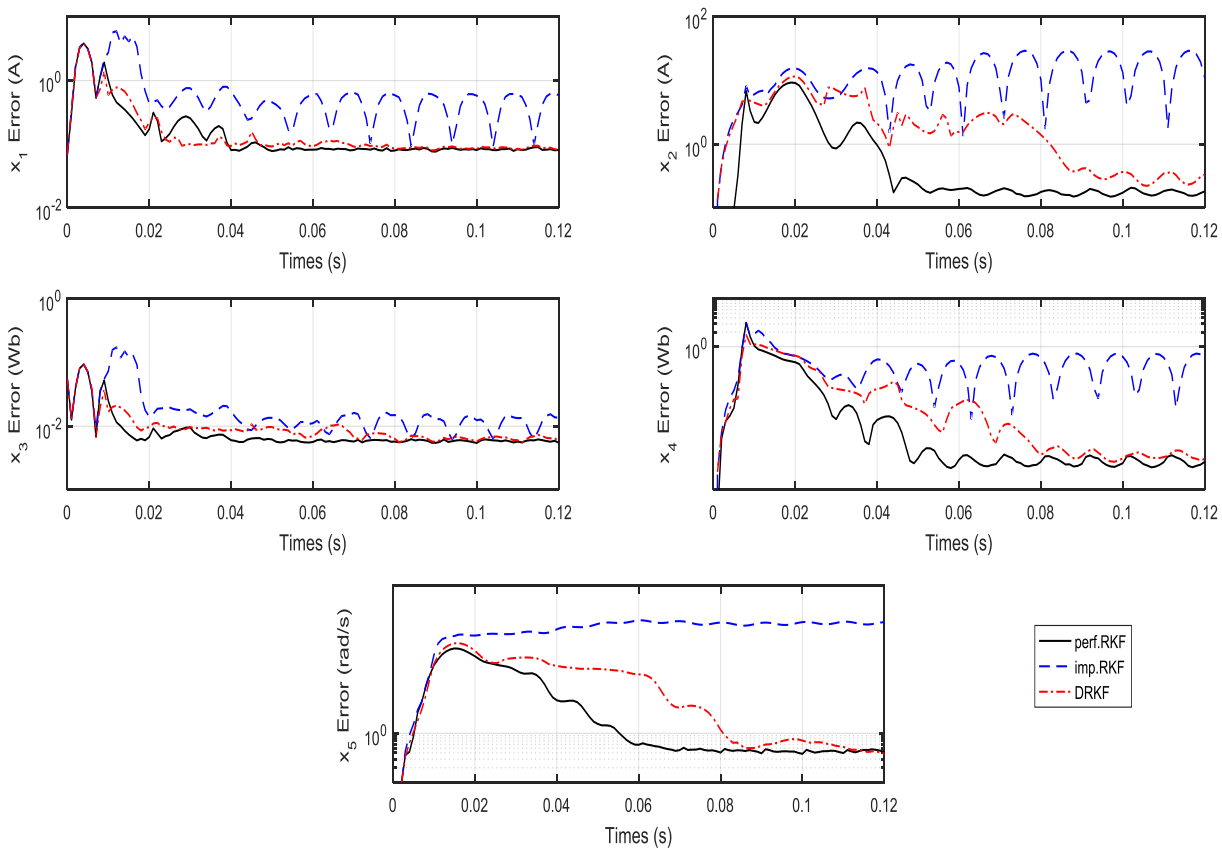

- perf.RKF
- - imp.RKF

Fig. 2. RMSE of states
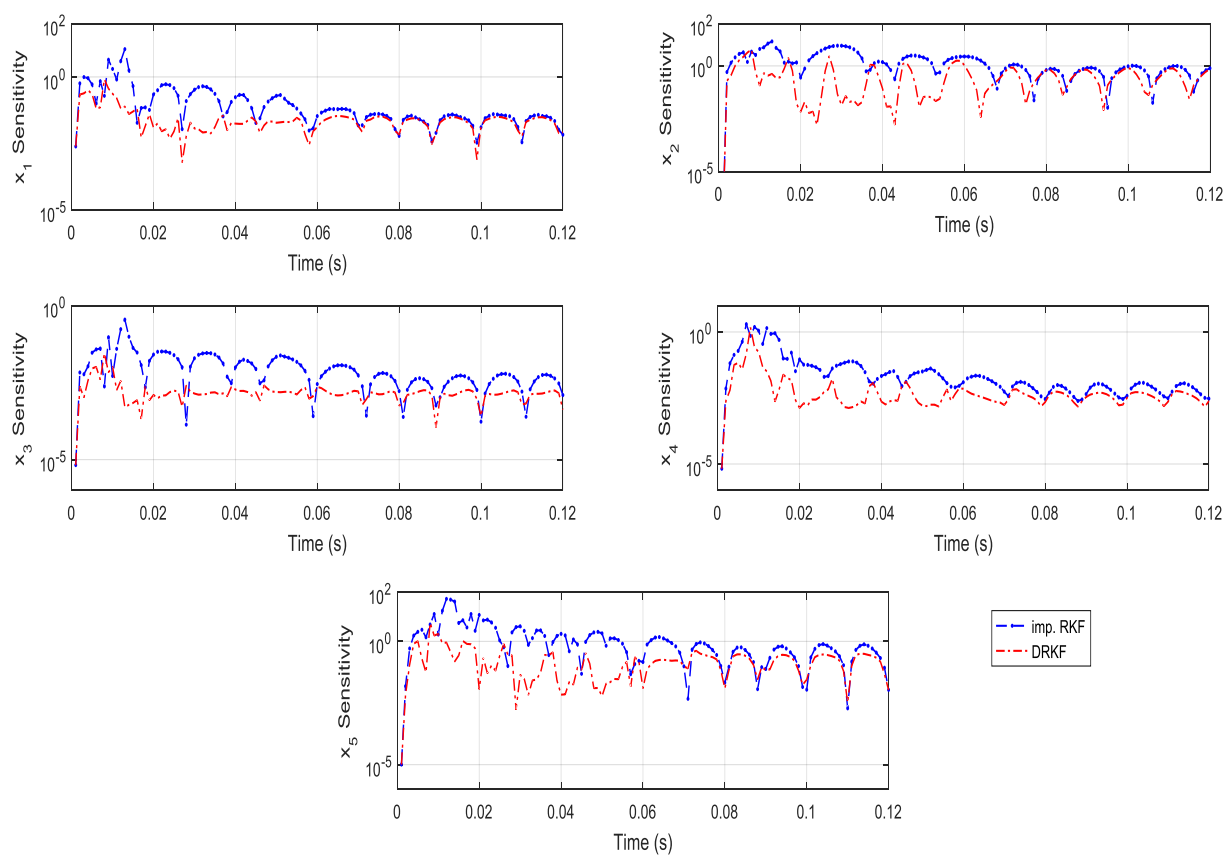

--- imp. RKF
---.DRKF

Fig. 3. States sensitivities with the uncertain stator resistance for case one 

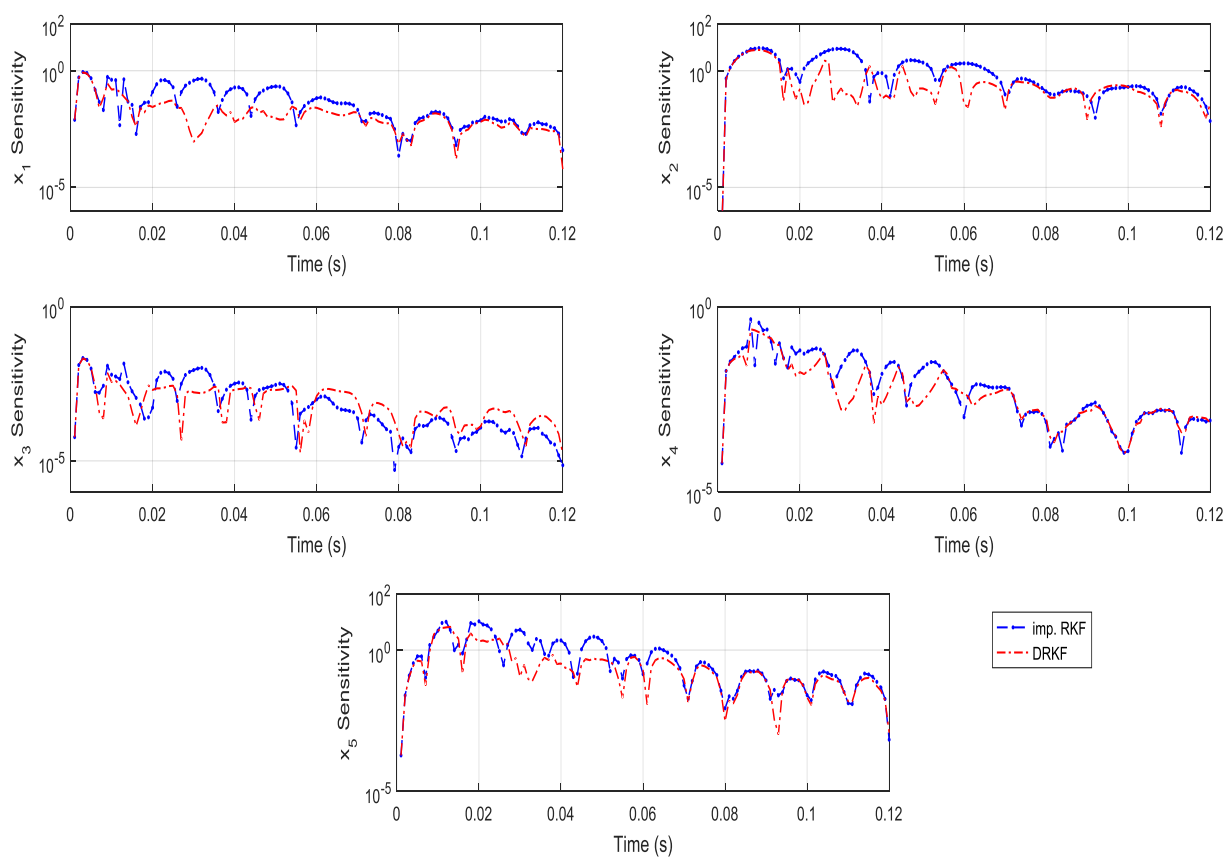

\begin{tabular}{l}
\hline-- imp. RKF \\
--- . DRKF \\
\hline
\end{tabular}

Fig. 4. States sensitivities with the uncertain rotor resistance for case one
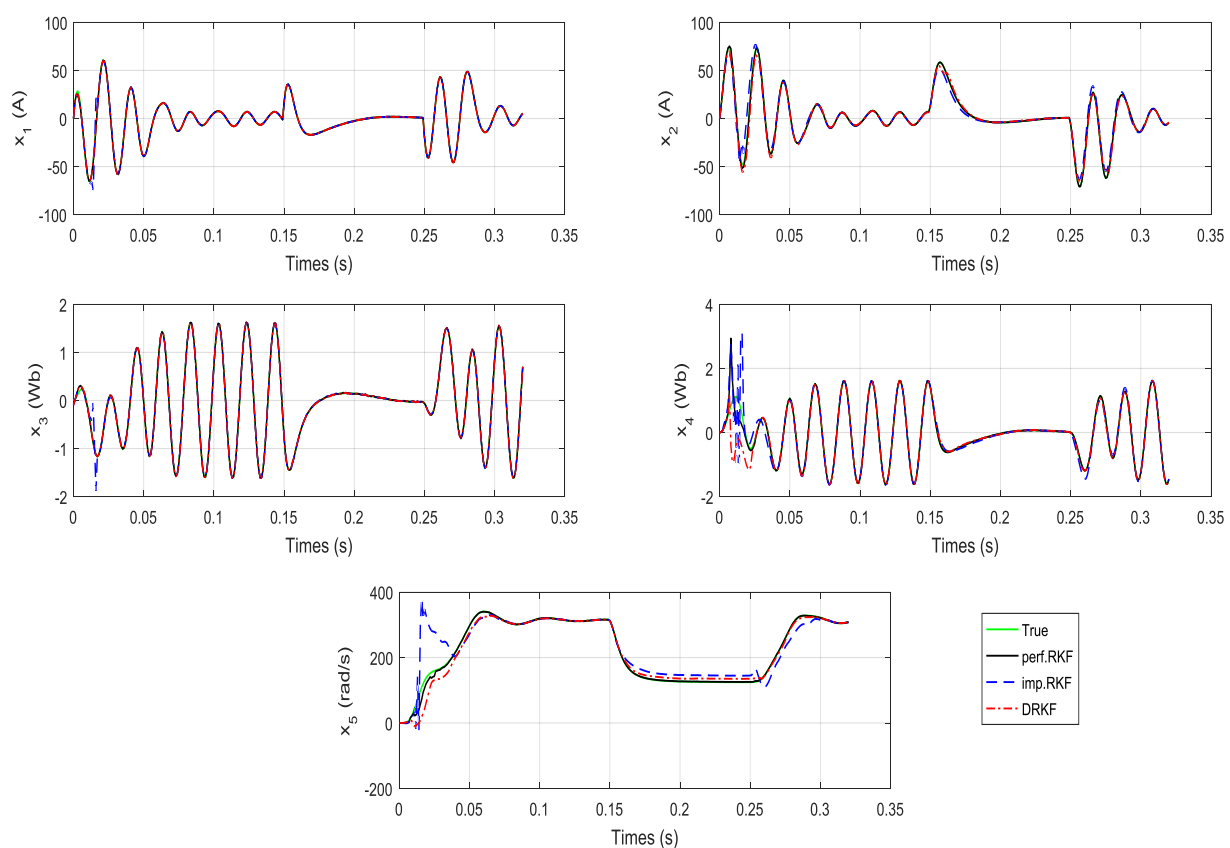

Fig. 5. True values and state estimations 

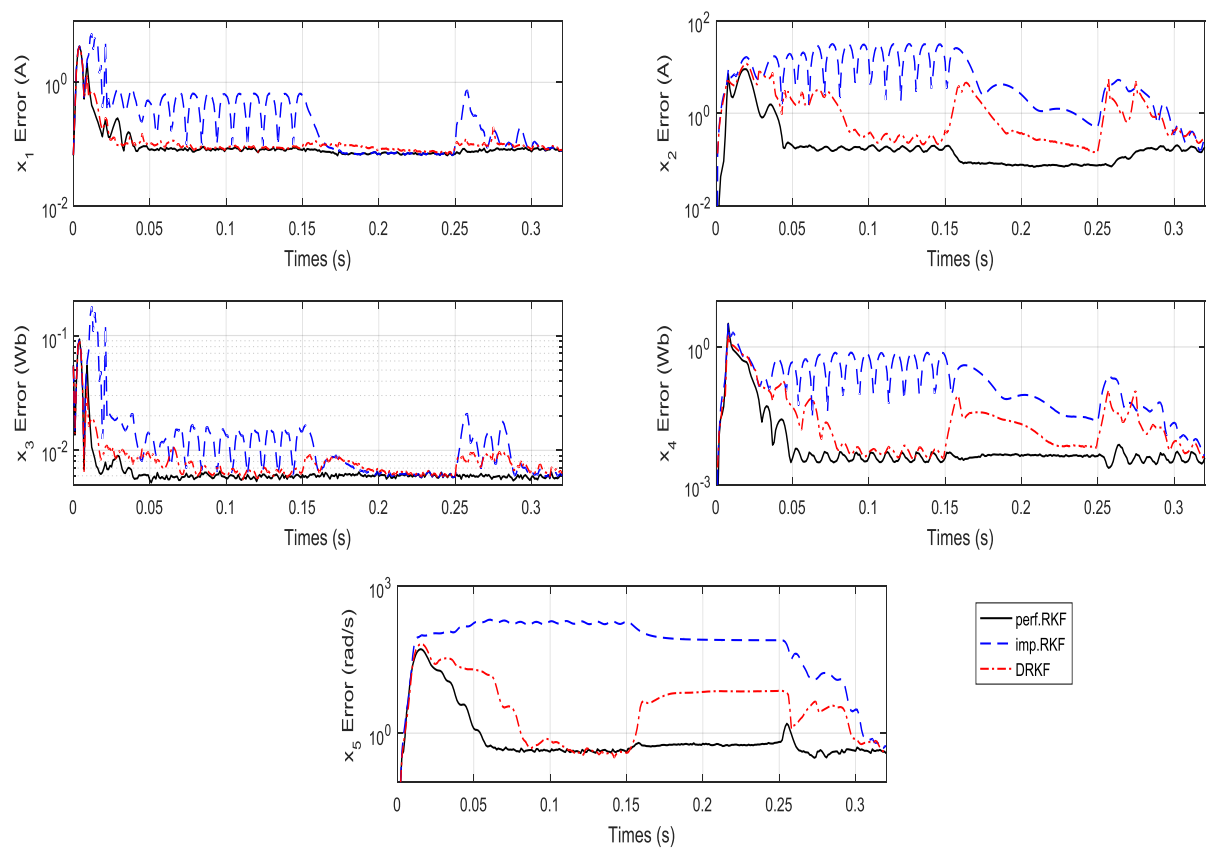

Fig. 6. RMSE of states

\section{Conclusion}

Based on rank sample rule, this paper introduces the desensitized optimal control technology into rank Kalman filter, and a robust desensitized rank Kalman filtering (DRKF) is proposed for nonlinear systems to mitigate the adverse effects of model parameter uncertainties. The sensitivity propagation of the deterministic sigma points is summarized, and a new desensitized cost function with a penalty function is designed to obtain the suboptimal gain matrix. Then, an ideal no-load start at rated voltage and a three-phase sudden short circuit fault of the induction motor are simulated, in which the stator and rotor resistances have uncertainties. The simulation results show that the proposed DRKF algorithm has excellent performance and can track the motor speed.

\section{References}

1 Jafarzadeh, S., Lascu, C., Fadali, M.S.: 'State estimation of induction motor drives using the unscented Kalman filter', Ieee T Ind Electron, 2012, 59, (11), pp. 4207-4216

2 Borsjie, P., Chan, T.F., Wong, Y.K., Ho, S.L.: 'A comparative study of Kalman filtering for sensorless control of a Permanent-Magnet synchronous motor drive'. Proc. IEEE Int. Conf. Elect. Mach. Drives, San Antonio, 2005, pp. 815-822

3 Marino, R., Tomei, P., Verrelli, C.M.:'Induction motor control design'( Springer Science \& Business Media, 2010 edn.2010)

4 Crassidis, J.L., Junkins, J.L.:'Optimal estimation of dynamic systems'( Chapman \& HalllCRC Press, 2012, 2nd ed. edn.2012)

5 Arasaratnam, I., Haykin, S.: 'Cubature Kalman filters', Ieee T
Automat Contr, 2009, 54, (6), pp. 1254-1269

6 Simon, D.:'Optimal state estimation: Kalman, H infinity, and nonlinear approaches'( Wiley-Interscience, 2006 edn.2006)

7 Fu, H.M., Xiao, Q., Wu, Y.Z., Lou, T.S.: 'Rank Kalman filtering method', Journal of Mechanical Strength, 2014, 36, (04), pp. 521-526

8 Kim, Y., Sul, S., Park, M.: 'Speed sensorless vector control of induction motor using extended Kalman filter', Ieee T Ind Appl, 1994, 30, (5), pp. 1225-1233

9 Shi, K.L., Chan, T.F., Wong, Y.K., Ho, S.L.: 'Speed estimation of an induction motor drive using an optimized extended Kalman filter', Ieee T Ind Electron, 2002, 49, (1), pp. 124-133

10Barut, M., Bogosyan, S., Gokasan, M.: 'Speed-sensorless estimation for induction motors using extended Kalman filters', Ieee T Ind Electron, 2007, 54, (1), pp. 272-280

11Jafarzadeh, S., Lascu, C., Fadali, M.S.: 'Square root unscented Kalman filters for state estimation of induction motor drives', Ieee T Ind Appl, 2013, 49, (1), pp. 92-99

12Chan, T., Borsje, P., Wang, W. Application of Unscented Kalman filter to sensorless permanent-magnet synchronous motor drive. in Electric Machines and Drives Conference, 2009. IEMDC'09. IEEE International. 2009: IEEE.

13Gopinath, G.R., Das, S.P. A cubature Kalman filter based speed and position estimator for permanent magnet synchronous motor. in 2015 IEEE Symposium on Sensorless Control for Electrical Drives (SLED), June 2015. 2015: IEEE.

14Lou, T., Fu, H., Zhang, Y., Wang, Z.: 'Consider unobservable uncertain parameters using radio beacon navigation during Mars entry', Adv Space Res, 2015, 55, (4), pp. $1038-1050$

15Lou, T., Wang, L., Su, H., et al.: 'Desensitized cubature 
Kalman filter with uncertain parameters', Journal of the Franklin Institute, 2017, 354, (18), pp. 8358 - 8373

16Karlgaard, C.D., Shen, H.J.: 'Desensitized Kalman filtering', IET Radar, Sonar \& Navigation, 2013, 7, (1), pp. 2-9

17Lou, T., Liu, J., Jin, P., Wang, Y.: 'MACV/Radio integrated navigation for Mars powered descent via robust desensitized central difference Kalman filter', Adv Space Res, 2017, 59, (1), pp. 457-471

18Shen, H., Karlgaard, C.D.: 'Sensitivity reduction of unscented Kalman filter about parameter uncertainties', IET Radar, Sonar \& Navigation, 2015, 9, (4), pp. 374-383

19Lou, T., Wang, L., Su, H., et al.: 'Desensitized cubature Kalman filter with uncertain parameters', Journal of the Franklin Institute, 2017, 354, (18), pp. 8358-8373

20 Yang, X., Liu, G., Chen, N., Lou, T.: 'Desensitized ensemble Kalman filtering for induction motor estimation', Ieee Access, 2019, 7, pp. 78029-78036

21Lou, T.: 'Desensitized Kalman filtering with analytical gain', arXiv: 1504.04916, 2015

22Lou, T.: 'Consider Uncertain Parameters based on Sensitivity Matrix', arXiv preprint arXiv:1503.08379, 2015

23Ishihara, S., Yamakita, M.: 'Adaptive robust UKF for nonlinear systems with parameter uncertainties'. Industrial Electronics Society, IECON 2016-42nd Annual Conference of the IEEE, Florence, Italy, Oct. 2016, 2016, pp. 48-53

24Wang, L., Xia, Y.: 'Mars entry navigation with uncertain parameters based on desensitized extended Kalman filter', Ieee T Ind Inform, 2015, 11, (5), pp. 998-1005

25Karlgaard, C.D., Shen, H.J.: 'Robust state estimation using desensitized divided difference filter', Isa T, 2013, 52, (5), pp. 629-637

26Fu, H.M., Xiao, Q., Lou, T.S., Xiao, M.L.: 'Nonlinear and non-Gaussian rank filter method[j]', Journal of Aerospace Power, 2015, 30, (10), pp. 2318-2322 